\title{
CONSIDERATION OF UNCERTAINTIES IN THE PRELIMINARY DESIGN CASE OF AN ELECTROMAGNETIC SPINDLE
}

\author{
Hassen Trabelsi, Amir Guizani, Dhouha Tounsi \\ Mechanics, Modelling and Production Research Laboratory, National School of Engineers of Sfax, University of Sfax, \\ Sfax, Tunisia; e-mail: hassen.trabelsi@outlook.fr \\ MONCEF HAMMADI \\ QUARTZ EA7393, Supméca-Paris, Saint-Ouen, France
}

Maher Barkallah, Mohamed Haddar

Mechanics, Modelling and Production Research Laboratory, National School of Engineers of Sfax, University of Sfax, Sfax, Tunisia

\begin{abstract}
Modeling and evaluation of uncertainties constitute indeed one of the key points when making any decision. For this, designers have to compare the measured or calculated value with a range of permissible values in order to obtain a guaranteed design process. Thus, in this work, simulation of the dynamic behavior of an electromagnetic spindle was done based on the interval computation technique. Indeed, the use of this technique makes it possible to obtain a set of values for different design parameters of the spindle and, consequently, to avoid making several simulations which could make the system useless, expensive or ineffective. The proposed model is based on the combination of Matlab with ModelCenter. Matlab was used to model and simulate the system and ModelCenter to perform parametric studies to verify the influences of uncertainty on the dynamic behavior of the electromagnetic spindle and to determine the optimal design parameters.
\end{abstract}

Keywords: preliminary design, electromagnetic spindle, dimensioning, interval computation, simulation, optimization

\section{Introduction}

The preliminary design is upstream of the designing process of a mechatronic system. From the set of requirements, it consists in determining a set of possible solutions in a very large research space, and structured by partial and uncertain knowledge of the future system and its environment. Mechatronic systems (Guizani et al., 2017) face different physical constraints such as vibration and shocks (Makowski and Zalewski, 2015) that are directly related to reliability of the system. Thus, a good preliminary design (Colton and Ouellette, 1994) of such a system is required to minimize harmful vibrations. Conventional design methodologies based on a loop "design-simulate-back at the initial stage in case of failure" (Teorey et al., 1986) appear to be increasingly obsolete. During the past years, many studies have been conducted to optimize design (He and McPhee, 2005; Affi et al., 2007), more precisely at the pre-sizing step (Amendola et al., 2017). This step is often expensive and requires higher computation times in order to obtain the optimal values of design parameters. In this work, we propose using interval computation (Alefeld and Mayer, 2000; Hansen and Walster, 2003; Trabelsi et al., 2015) to simulate the dynamic behavior of an electromagnetic spindle (Hentati et al., 2013; Bouaziz et al., 2016). It provides rigorous evaluation that allows designing a mechatronic system while minimizing the number of simulations and, consequently, the calculation time. So, theoretically and using the interval computation, we can obtain the system response by intervals and frame the solution 
space. However, the current problem is that the evaluation of this method coupled with Newmark and Newton Raphson (Faroughi and Lee, 2015) and (Gościniak and Gdawiec, 2019) to determine the dynamic response has not yet been tested on a scalable example. Therefore, the main objective of this work is to evaluate the effectiveness of the interval simulation method to simulate the dynamic behavior of an electromagnetic spindle by intervals. Then, to determine the optimal design parameters of the spindle. With the uncertainties introduced to some designs parameters, the designer can make a good idea of the variation effects of these variables on the dynamic behavior of the electromagnetic spindle. Furthermore, simulation with intervals can help one to make a good decision about the best values of design parameters.

The paper is organized as follows: after Introduction, Section 2 details the dynamic model of the electromagnetic spindle. Section 3 is dedicated to presentation of the dynamic behavior by intervals as a guaranteed method that can encompass all the solutions. In Section 4, a parametric study is done to some parameters to show that the uncertainty influences the dynamic behavior of the electromagnetic spindle. Section 5 of this paper is devoted to the optimization of the spindle concept. Finally, conclusions are drawn in Section 6. So, in the next parts of this paper, the dynamic behavior of the electromagnetic spindle is studied using the interval computation method. The goal is to obtain a set of solutions for various design variables of the spindle in order to achieve a guaranteed design process. The proposed study is based on the combination of Matlab with ModelCenter. Matlab is used to model and simulate the dynamic behavior of the spindle, and ModelCenter to verify the influences of the uncertainty and to determine the optimal design solutions.

\section{Dynamic behavior of an electromagnetic spindle}

In high speed machining, the spindle is considered as the main element of the machining system. In fact, it ensures rotation of the tool/tool holder at high rotation speeds and guarantees the compatibility between the torque and the metal cutting. During recent years, the spindles supported by electromagnetic bearings have become the most commonly used. Indeed, those last are supposed the most efficient to ensure the best performance of the spindle supported without any mechanical contact.

The electromagnetic bearings advantages are: absence of lubrication, high rotational speed, high rigidity, and low vibration. Compared with rolling bearings, Knospe (2007), Kimman et al. (2010) and Gourc et al. (2011) deduced that the electromagnetic bearings have a long life, a high robustness to shock caused by accidental forces, and a high rotational speed with minimum energy dissipation.

This study is based on the dynamic model of an electromagnetic spindle system (Bouaziz et al., 2016) shown in Fig. 1. The electromagnetic spindle studied is considered as a mechatronic system that consists of a spindle body (shaft or rotor), two electromagnetic bearings placed at the bottom and at the top, and an axial stop. The global equation of motion is formulated by applying Lagrange's formalism to the kinetic and potential energy expressions of the shaft. It is written as follows (Hentati et al., 2013; Bouaziz et al., 2016)

$$
\mathbf{M} \ddot{\mathbf{Q}}+\left(\mathbf{G}+\mathbf{D}+\mathbf{C}_{b}(t)\right) \dot{\mathbf{Q}}+\left(\mathbf{K}+\mathbf{K}_{b}(t)\right) \mathbf{Q}=\mathbf{F}_{c(x, y, z)}(t, \mathbf{Q})
$$

The displacement vector containing the degrees of freedom of elastic movement and those of rigid motion is expressed by

$$
\mathbf{Q}=\left[U_{1}, V_{1}, W_{1}, \theta_{x 1}, \theta_{y 1}, \theta_{z 1}, \ldots, U_{i}, V_{i}, W_{i}, \theta_{x i}, \theta_{y i}, \theta_{z i}, X_{A}, Y_{A}, Z_{A}, \alpha_{x}, \alpha_{y}, \alpha_{z}\right]^{\mathrm{T}}
$$

where $\mathbf{M}$ is the mass matrix, $\mathbf{G}$ is the gyroscopic matrix, and $\mathbf{K}$ is the dynamic stiffness matrix. $\mathbf{D}$ is the modal damping matrix, $\mathbf{K}_{b}(t)$ and $\mathbf{C}_{b}(t)$ are variable matrices containing the coeffi- 


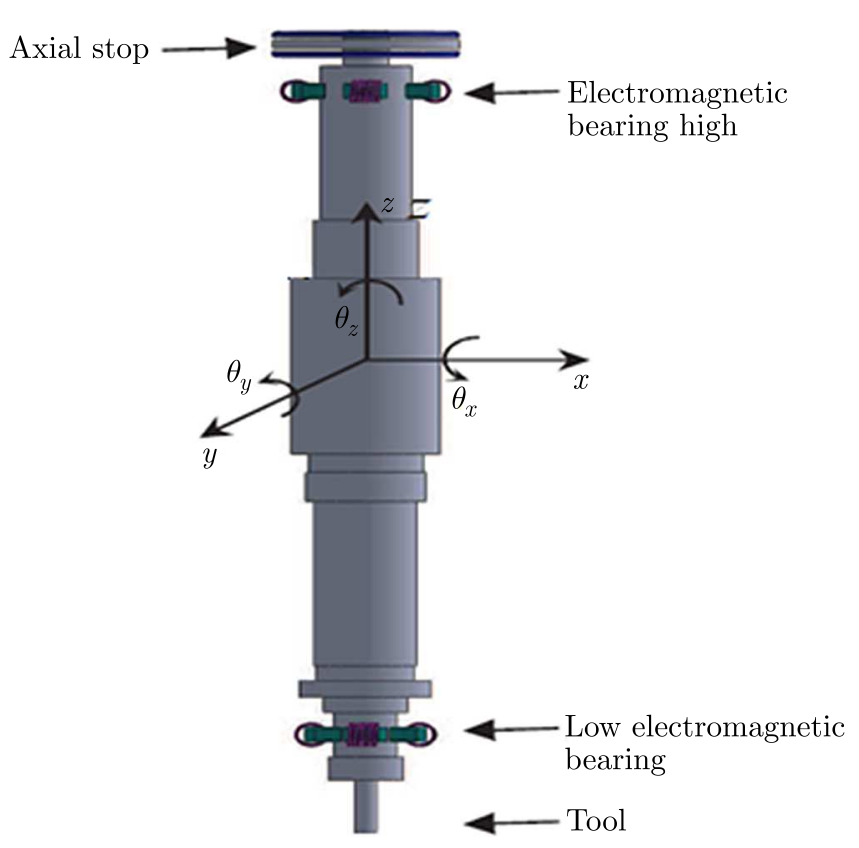

Fig. 1. Electromagnetic spindle

cients of stiffness of the electromagnetic bearings. The variables of the electromagnetic spindle previously mentioned are defined as follows

$$
\begin{aligned}
& \mathbf{M}=\left[\begin{array}{cc}
\mathbf{M}_{F} & \mathbf{M}_{R F} \\
\mathbf{M}_{R F}^{\mathrm{T}} & \mathbf{M}_{R}
\end{array}\right] \quad \mathbf{G}=2 \Omega\left[\begin{array}{cc}
\mathbf{G}_{F} & \mathbf{G}_{R F} \\
-\mathbf{G}_{R F}^{\mathrm{T}} & \mathbf{G}_{R}
\end{array}\right] \\
& \mathbf{K}=\left[\begin{array}{cc}
\mathbf{K}_{F} & \mathbf{0} \\
\mathbf{0} & \mathbf{0}
\end{array}\right]-\Omega^{2}\left[\begin{array}{cc}
\mathbf{C}_{F} & \mathbf{0} \\
\mathbf{0} & \mathbf{0}
\end{array}\right]
\end{aligned}
$$

In addition, $\mathbf{C}_{F}$ is the centrifugal force, $F$ and $R$, respectively, designate the two flexible and rigid movements

$$
\mathbf{D}=\alpha \mathbf{M}+\beta \mathbf{K}
$$

where $\alpha$ and $\beta$ are the damping coefficients (Bouaziz et al., 2016)

$$
\begin{aligned}
\mathbf{K}_{b}(t) & =\left[\begin{array}{ccccccc}
0 & \cdot & \cdot & 0 & \cdot & \cdot & 0 \\
\cdot & \cdot & \cdot & \cdot & \cdot & \cdot & 0 \\
\cdot & K_{x x} & K_{x y} & \cdot & \cdot & \cdot & \cdot \\
0 & K_{y x} & K_{y y} & \cdot & \cdot & \cdot & \cdot \\
\cdot & \cdot & \cdot & 0 & K_{x x} & K_{y y} & \cdot \\
\cdot & \cdot & \cdot & \cdot & K_{y x} & K_{y y} & 0 \\
\cdot & \cdot & \cdot & \cdot & 0 & \cdot & 0
\end{array}\right] \\
\mathbf{C}_{b}(t) & =\left[\begin{array}{ccccccc}
0 & \cdot & \cdot & 0 & \cdot & \cdot & 0 \\
\cdot & \cdot & \cdot & \cdot & \cdot & \cdot & 0 \\
\cdot & C_{x x} & C_{x y} & \cdot & \cdot & \cdot & \cdot \\
0 & C_{y x} & C_{y y} & \cdot & \cdot & \cdot & \cdot \\
\cdot & \cdot & \cdot & 0 & C_{x x} & C_{y y} & \cdot \\
\cdot & \cdot & \cdot & \cdot & C_{y x} & C_{y y} & 0 \\
\cdot & \cdot & \cdot & \cdot & 0 & \cdot & 0
\end{array}\right]
\end{aligned}
$$




\section{Shaft modeling approach}

$$
\begin{aligned}
E_{c} & =\frac{1}{2} \rho A \int_{0}^{L}\left[\left(\dot{X}_{A}^{2}+\dot{Y}_{A}^{2}+\dot{Z}_{A}^{2}\right)+\left(\dot{U}^{2}+\dot{V}^{2}+\dot{W}^{2}\right)+2\left(\dot{U}_{Y_{A}}+\dot{V} \dot{Z}_{A}\right)\right] d z \\
& +\frac{1}{2} \rho I_{m} \int_{0}^{L}\left[\left(\dot{\alpha}_{x}^{2}+\dot{\alpha}_{y}^{2}+\dot{\alpha}_{z}^{2}\right)+\left(\dot{\theta}_{x}^{2}+\dot{\theta}_{y}^{2}+\dot{\theta}_{z}^{2}\right)+4 \Omega\left(\dot{\alpha}_{y} \alpha_{z}\right)+4 \Omega\left(\dot{\theta}_{y} \theta_{z}\right)\right. \\
& \left.+4 \Omega\left(\dot{\alpha}_{y} \theta_{z}-\dot{\alpha}_{z} \theta_{y}\right)+2\left(\dot{\alpha}_{y} \dot{\theta}_{y}+\dot{\alpha}_{z} \dot{\theta}_{z}\right)+2 \Omega^{2}\right] d z \\
E_{p} & =\frac{1}{2} \int_{0}^{l} E I\left(\dot{U}_{b}^{2}+\dot{V}_{b}^{2}+\dot{W}_{b}^{2}\right) d x+\frac{1}{2} \int_{0}^{l} K G A\left(\dot{U}_{s}^{2}+\dot{V}_{s}^{2}+\dot{W}_{s}^{2}\right) d z
\end{aligned}
$$

with $\left\{U, V, W, \theta_{x}, \theta_{y}, \theta_{z}\right\}$ and $\left\{\dot{U}, \dot{V}, \dot{W}, \dot{\theta}_{x}, \dot{\theta}_{y}, \dot{\theta}_{z}\right\}$ are, respectively, the displacements and velocities corresponding to the elastic movements, $\left\{X_{A}, Y_{A}, Z_{A}, \alpha_{x}, \alpha_{y}, \alpha_{z}\right\}$ and $\left\{\dot{X}_{A}, \dot{Y}_{A}, \dot{Z}_{A}, \dot{\alpha}_{x}, \dot{\alpha}_{y}, \dot{\alpha}_{z}\right\}$ are the displacements and velocities relative to the rigid movements; $\mathbf{F}_{c(x, y, z)}(t, \mathbf{Q})$ is the vector of the next cutting effort $x, y$ and $z$.

The displacements $\{U, V, W\}$ are composed of the displacements $\left\{U_{b}, V_{b}, W_{b}\right\}$ due to the effect of bending and displacements $\left\{U_{s}, V_{s}, W_{s}\right\}$, which result from the shear effect of the element.

$E$ and $I$ represent, respectively, Young's modulus and the moment of inertia, $K$ and $G$, respectively, show the shear coefficient and the shear modulus, $A$ is the section of the element. $K$ and $G$, respectively, are the shear coefficient and the shear modulus.

\section{Modeling of electromagnetic bearings}

$$
F_{p x}=\sum_{k=1}^{n} F_{p k} \cos \varphi_{k} \quad F_{p y}=\sum_{k=1}^{n} F_{p k} \sin \varphi_{k}
$$

The components of the force exerted by the electromagnetic bearings in the $x$ - and $y$-directions, for a bearing with four electromagnets, can be rewritten as in the following

$$
\begin{aligned}
& F_{p x 4}=a\left[\left(\frac{1-\frac{k_{p} u_{x}}{I_{0}}-\frac{k_{d} \dot{u}_{x}}{I_{0}}}{1-\frac{u_{x}}{e_{0}}}\right)^{2}-\left(\frac{1+\frac{k_{p} u_{x}}{I_{0}}+\frac{k_{d} \dot{u}_{x}}{I_{0}}}{1+\frac{u_{x}}{e_{0}}}\right)^{2}\right] \\
& F_{p y 4}=a\left[\left(\frac{1-\frac{k_{p} u_{y}}{I_{0}}-\frac{k_{d} \dot{u}_{y}}{I_{0}}}{1-\frac{u_{y}}{e_{0}}}\right)^{2}-\left(\frac{1+\frac{k_{p} u_{y}}{I_{0}}+\frac{k_{d} \dot{u}_{y}}{I_{0}}}{1+\frac{u_{y}}{e_{0}}}\right)^{2}\right]
\end{aligned}
$$

where $e_{0}$ is the nominal airgap

$$
I_{i}=k_{p} u_{i}+k_{d} \dot{u}_{i} \quad i=x, y
$$

$k_{p}$ and $k_{d}$ present the proportional gain and the derivative gain, successively

$$
k_{p}=k_{0}+k_{1} \cos w t+k_{2} \cos 2 w t
$$

$k_{0}$ and $k_{2}$ are constants relative to the controller, and $w$ is the angular velocity

$$
\begin{aligned}
& \left\{\begin{array}{l}
F_{p x} \\
F_{p y}
\end{array}\right\}=\mathbf{K}\left\{\begin{array}{l}
u_{x} \\
u_{y}
\end{array}\right\}+\mathbf{C}\left\{\begin{array}{l}
\dot{u}_{x} \\
\dot{u}_{y}
\end{array}\right\} \\
& \mathbf{K}=\left[\begin{array}{ll}
K_{x x} & K_{x y} \\
K_{y x} & K_{y y}
\end{array}\right] \\
& \quad \mathbf{C}=\left[\begin{array}{ll}
C_{x x} & C_{x y} \\
C_{y x} & C_{y y}
\end{array}\right]
\end{aligned}
$$




\section{Axial thrust force}

The axial magnetic abutment is

$$
F_{\text {thrust }}=K_{i z} I_{z}+K_{z} u_{z}
$$

\section{Predictive model of the cutting force in peripheral milling}

The expressions of these three components are as follows

$$
F_{t}=K_{t} a_{p} h\left(\Phi_{j}(t)\right) \quad F_{r}=K_{r} F_{t} \quad F_{a}=K_{a} F_{t}
$$

$\Phi_{j}(t)$ is the angle of rotation of the $j$-th tooth

$$
\Phi_{j}(t)=w t+j \Phi_{p} \quad j=0,1, \ldots, Z-1
$$

$w$ and $\Phi_{p}$ are, respectively, the angular velocity and the angle between two successive edges. $a_{p}, K_{t}, K_{r}$ and $K_{a}$ are, respectively, the axial cutting depth and the specific cutting coefficients

$$
\begin{aligned}
& h\left(\Phi_{j}(t)\right)=h_{s}\left(\Phi_{j}(t)\right)+h_{d}\left(\Phi_{j}(t)\right) \quad h_{s}\left(\Phi_{j}(t)\right)=f_{z} \sin \Phi_{j}(t) \\
& h_{d}\left(\Phi_{j}(t)\right)=\left[u_{x}(t)-u_{x}(t-\tau)\right] \sin \Phi_{j}(t)-\left[u_{y}(t)-u_{y}(t-\tau)\right] \cos \Phi_{j}(t) \\
& \tau=\frac{60}{\Omega Z}
\end{aligned}
$$

$U_{x}(t)$ and $U_{y}(t)$ present the deflections of the tool along the directions $x$ and $y$ at the present time. $U_{x}(t-\tau)$ and $U_{y}(t-\tau)$ are the deflections along the directions $x$ and $y$ at the previous time, $\Phi$ is the period of tooth passage. The values of the different parameters used in the analysis of the studied model are given in Tables 1 and 2.

Table 1. Characteristics of the electro-spindle

\begin{tabular}{|c|l|c|c|}
\hline Variables & \multicolumn{1}{|c|}{ Nomenclature } & Values & Units \\
\hline \hline$\Omega$ & Rotation speed of the spindle & 20000 & $\mathrm{tr} / \mathrm{min}$ \\
\hline$L$ & Length of the shaft & 651.95 & $\mathrm{~mm}$ \\
\hline$I_{r}$ & Moment of inertia & 0.11 & $\mathrm{~kg} \cdot \mathrm{m}^{2}$ \\
\hline$m$ & Spindle mass & 16.03 & $\mathrm{~kg}$ \\
\hline$E$ & Young's modulus & $2.11 \cdot 10^{11}$ & $\mathrm{~Pa}$ \\
\hline$\rho$ & Density & 7.85 & $\mathrm{~g} / \mathrm{cm}^{3}$ \\
\hline
\end{tabular}

The stiffness coefficients and the damping coefficients $K_{i j}$ and $C_{i j}$ for all $(i, j) \in(x, y)$ depend on time, so their measured values are determined during simulation in Matlab.

\section{Simulation by interval of the dynamic behavior of the electromagnetic spindle}

In this Section, the evaluation of the dynamic behavior of the electromagnetic spindle system is studied with uncertainties. The uncertainties are applied to the values of the design parameters that define the dynamic model of the system. The evaluation of Newmark's method with the Newton Raphson (Faroughi and Lee, 2015) and (Gościniak and Gdawiec, 2019) with uncertainties in real cases provided disappointing results, often divergent. However, the choice of parameters represented by intervals acts on the divergence of the model (Alefeld and Mayer, 2000) and (Trabelsi et al., 2015). The main advantage is to obtain convergent results, which reflect the efficiency of this method. So, the system of differential equations is solved with the interval simulation method. The uncertainty was introduced in the reference values of the forces 
Table 2. Parameters of electromagnetic bearings

\begin{tabular}{|c|l|c|c|}
\hline Variables & \multicolumn{1}{|c|}{ Nomenclature } & Values & Units \\
\hline \hline$\mu_{0}$ & Vacuum permeability & $4 \pi \cdot 10^{-7}$ & $\mathrm{~Wb} / \mathrm{Am}$ \\
\hline$e_{0}$ & Nominal air gap & 0.8 & $\mathrm{~mm}$ \\
\hline$S$ & Section of the electromagnet & 1.2 & $\mathrm{~mm}^{2}$ \\
\hline$N_{e}$ & Number of windings & 300 & - \\
\hline$\theta_{e}$ & Electromagnetic recomposition angle & 22.5 & $\mathrm{deg}$ \\
\hline$I_{0}$ & Polarization current & 5 & $\mathrm{~A}$ \\
\hline$\alpha$ & Coefficient proportional to mass & $1 \cdot 10^{-5}$ & - \\
\hline$\beta$ & Coefficient proportional to stiffness & $1 \cdot 10^{-3}$ & - \\
\hline$K_{x x}, K_{x y}$ & Stiffness & - & $\mathrm{N} / \mathrm{m}$ \\
$K_{y y}, K_{y x}$ & coefficients & & $\mathrm{Ns} / \mathrm{m}$ \\
\hline$C_{x x}, C_{x y}$ & Damping & - & \\
$C_{y y}, C_{y x}$ & coefficients & & \\
\hline
\end{tabular}

of the bearings to determine the behavior of the system by intervals. In this study, the following uncertainties are considered:

- $5 \%$ on the derivative gain $k_{d}$

- $2 \%$ on the constant relative to the controller $k_{0}$

- $5 \%$ on the distance between the axial stop and the center of gravity $d_{b}$

- $2 \%$ on the diameter of element 1

- $2 \%$ on the length of element 1

Table 3. Design Parameters with uncertainty

\begin{tabular}{|c|c|c|c|c|}
\hline $\begin{array}{c}\text { Design } \\
\text { variables }\end{array}$ & $\begin{array}{c}\text { Average } \\
\text { value }\end{array}$ & $\begin{array}{c}\text { Uncertainty } \\
{[\%]}\end{array}$ & Interval & Units \\
\hline \hline$k_{d}$ & 42.4 & 5 & {$[40.09,44.31]$} & - \\
\hline$k_{0}$ & 4520 & 2 & {$[4429.6,4610.4]$} & - \\
\hline$d_{b}$ & 0.309 & 5 & {$[0.2935,0.3245]$} & $\mathrm{mm}$ \\
\hline$L_{1}$ & 38.1 & 2 & {$[37.338,38.862] \cdot 10^{-3}$} & $\mathrm{~mm}$ \\
\hline$D_{1}$ & 12.7 & 2 & {$[12.446,12.954] \cdot 10^{-3}$} & $\mathrm{~mm}$ \\
\hline
\end{tabular}

The calculation has been done in Matlab with the IntLab library. The temporal responses of the spindle are illustrated in Figs. 2 and 3. Therefore, with the Interval Based Simulation, we determined an envelope which includes a set of performance for the displacement $Z$ of the elastic part, as shown in Fig. 2. The movement of the tool is periodic with a period equal to the period of rotation.

The curve obtained in Fig. 3 represents the rotational movement $\theta_{x}$ of the elastic part of the electromagnetic spindle. According to those two figures, we obtained a similar result to that with fixed values. The obtained solutions are convergent and have the same appearance as the curve calculated with fixed values. Therefore, the good designs of the electromagnetic spindle have an influence on its response in order to minimize vibrations. Therefore, a simulation with uncertainty is necessary to make good sizing of the system. So, relying on this simulation, the designer can determine the solution that can fulfill the constraints imposed in the specifications document, which makes the sizing of the electromagnetic spindle system easier. 


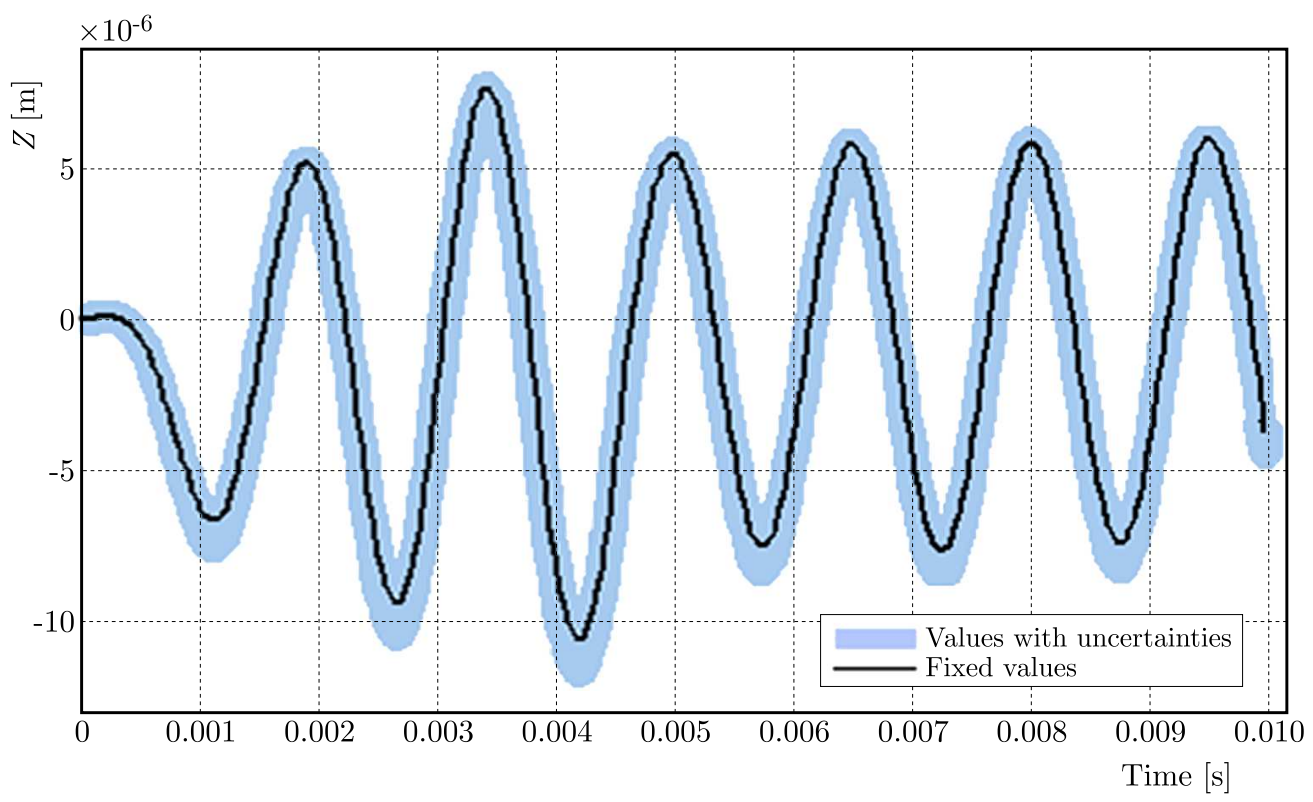

Fig. 2. Variation of displacement $Z$

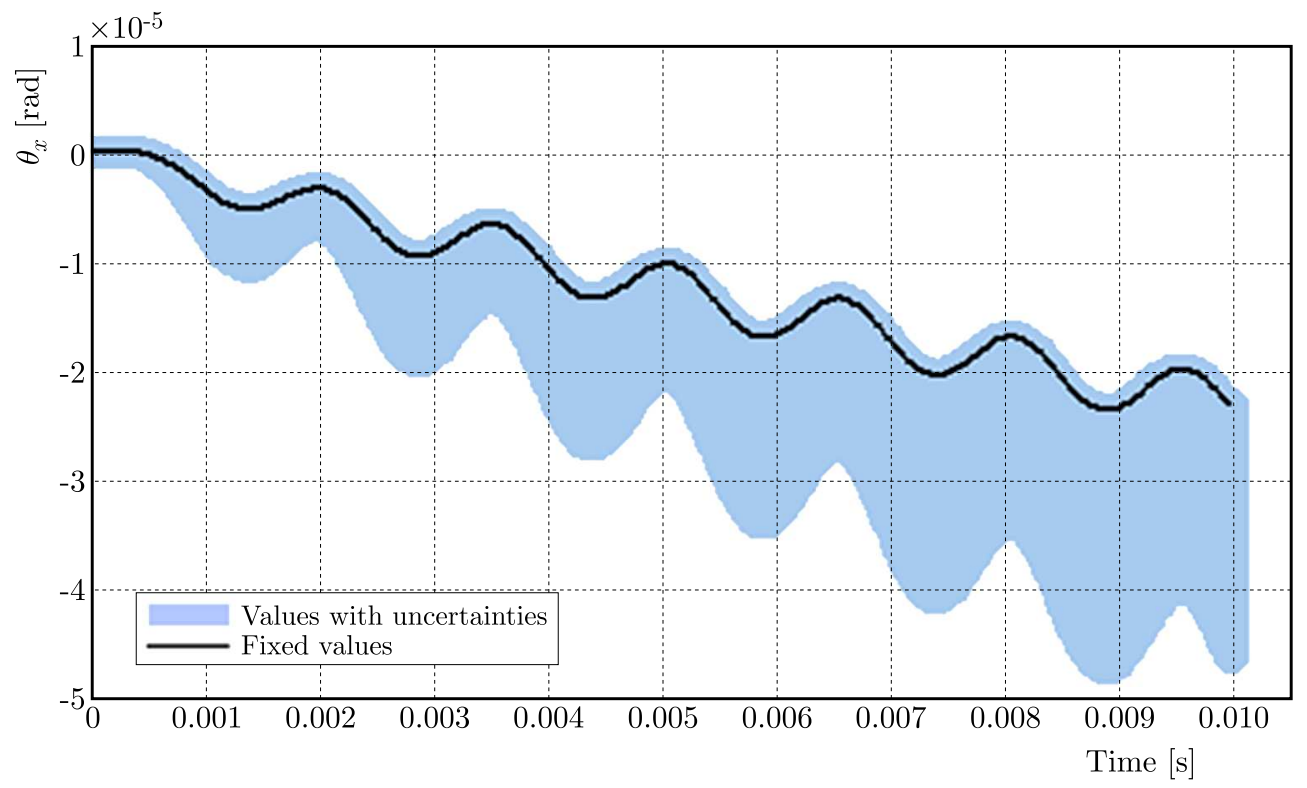

Fig. 3. Variation of rotational movement $\theta_{x}$

\section{Parametric studies and optimization of design variables}

\subsection{Parametric studies}

The objective of this part is to have a complete idea about the variation ranges of different design parameters that affect the dynamic behavior of the spindle.

This study is very useful for limiting the search space of variables that do not have a great influence on the behavior of the system. The curves shown in Fig. 5 represent the maximum displacements $\left(Z\right.$ and $\theta_{x}$ ) of the spindle according to the design parameters $\left(k_{d}, k_{0}, d_{b}, L_{1}\right.$ and $D_{1}$ ). These curves are obtained thanks to the coupling between the model developed in Matlab and the ModelCenter multi-physics simulation tool (Vu et al., 2016). 

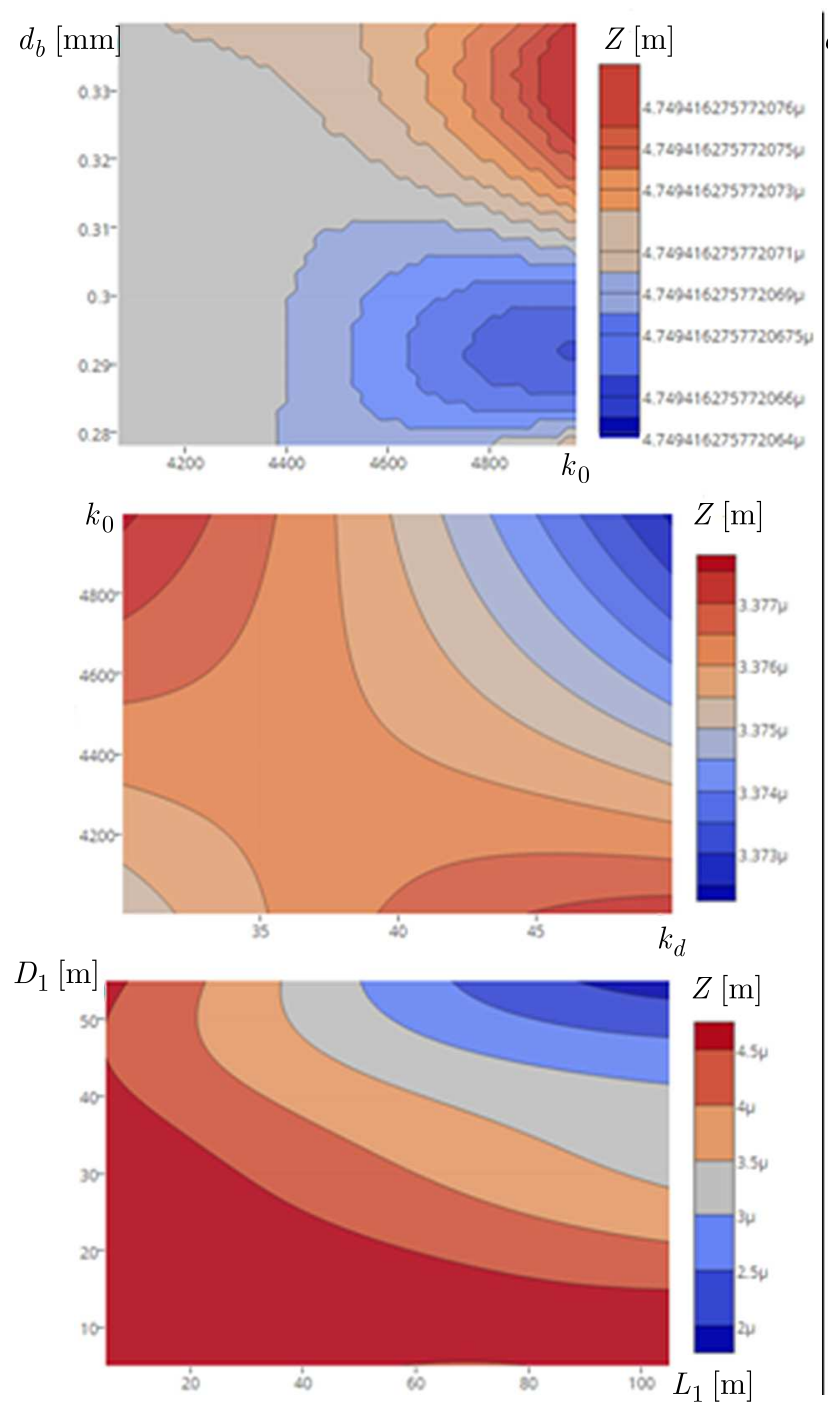
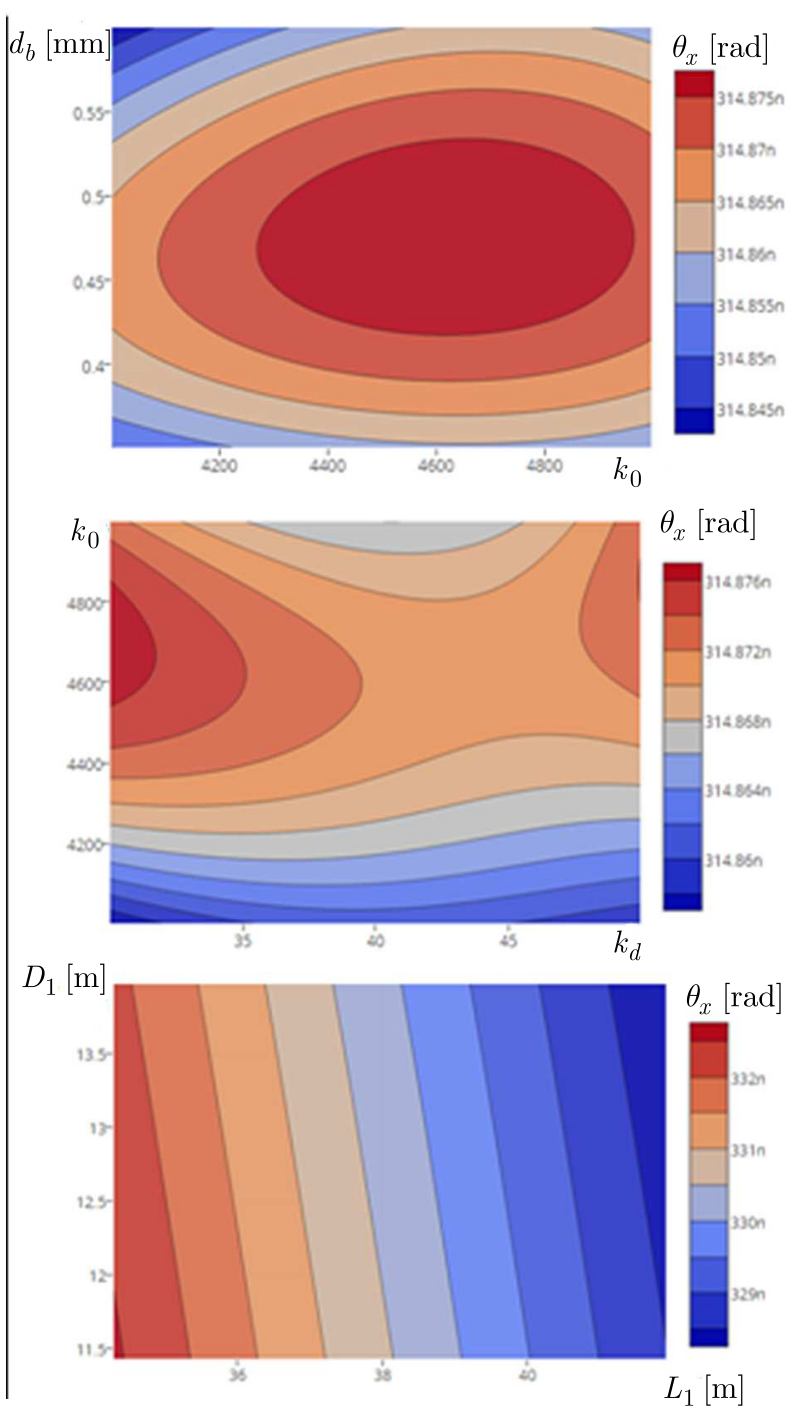

Fig. 4. Variation of displacements $Z$ and $\theta_{x}$

Each curve represents the variation of $Z$ and $\theta_{x}$ as a function of two parameters $\left[\left(d_{b}, k_{0}\right),\left(k_{0}, k_{d}\right),\left(D_{1}, L_{1}\right)\right]$. So, according to Fig. 4 , it is noticeable that the displacement $Z$ is maximum when $\left(d_{b}, k_{0}\right)$ are maximum, however, it is minimum when $\left(k_{0}, k_{d}\right)$ and $\left(D_{1}, L_{1}\right)$ are maximum.

For rotational movement $\theta_{x}$, it is maximum when $d_{b}$ is between $0.42 \mathrm{~mm}$ and $0.53 \mathrm{~mm}$, $k_{0}$ is between 4550 and 4850 and $k_{d}$ is between 0.3 and 0.32 . It is minimum when $\left(D_{1}, L_{1}\right)$ are maximum.

\subsection{Optimization of design variables}

In the following, an optimization study is performed according to the parameters $k_{d}, k_{0}, d_{b}$, $L_{1}$ and $D_{1}$. The objective of this study is to determine the optimum values in which $Z$ and $\theta_{x}$ displacements are minimum. The optimization is carried out using algorithms available in ModelCenter libraries, especially a Non-dominated Sorting Genetic Algorithm - NSGA II (Deb et al., 2002). NSGA II algorithm is adapted for multi-objective nonlinear optimizing problems. Instead of finding the best design, NSGA tries to find a set of best designs (e.g., Pareto set). A design is said to be dominated if there is another design that is superior to the design in all objectives. According to Fig. 5, we obtained eight optimal solutions. Each point of the Pareto- 
-Front is characterized by the input vector (design parameters to optimize) and the output vector (objective functions to be achieved). After the generation of the Pareto front, the best design solutions are summarized in Table 4.

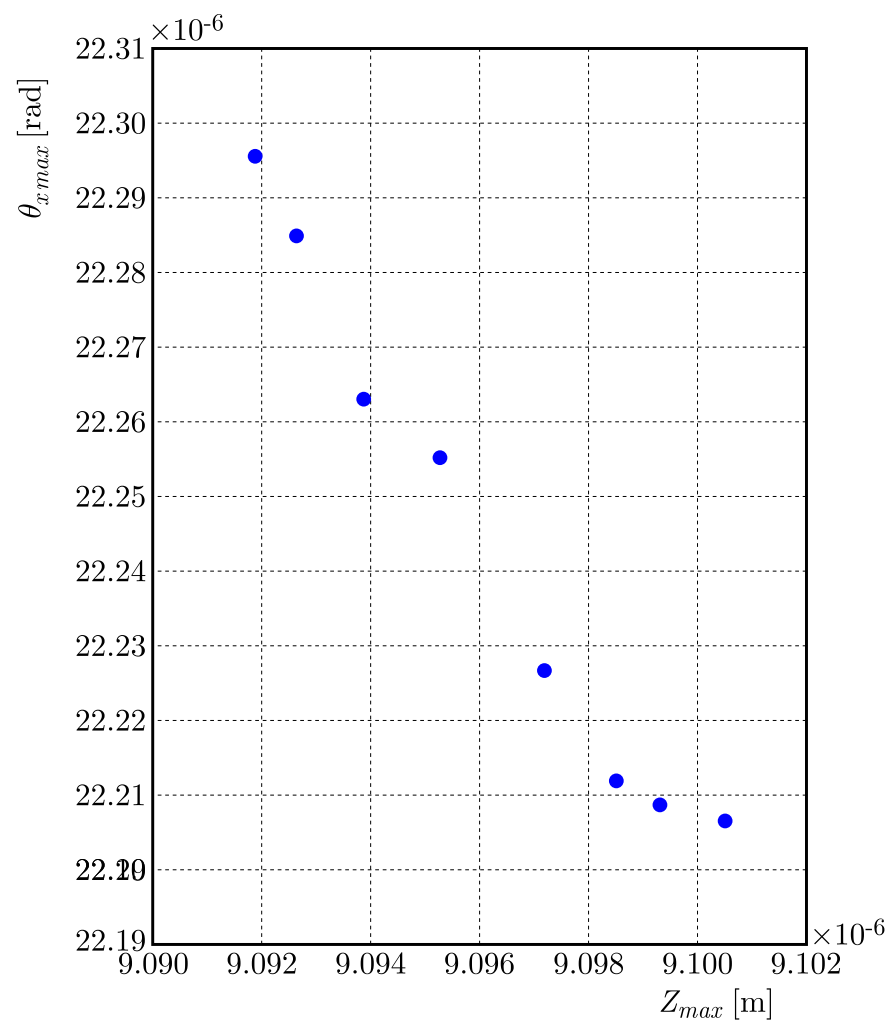

Fig. 5. Pareto-Front of the electromagnetic spindle

The values presented in the Pareto-Front of Fig. 5 show that the variation between the different solutions obtained is low, which is due to the fact:

- That the percentages of uncertainties chosen (which reflect the values domain of the design variables $k_{d}, k_{0}, d_{b}, L_{1}$ and $D_{1}$ ) are low, which are between $2 \%$ and $5 \%$.

- In addition, to get proper functioning of the electromagnetic spindle, the displacements $Z$ and $\theta_{x}$ should be small, since our objective is to minimize the vibrations according to the design parameters mentioned before.

Table 4. Optimal design parameters of the spindle

\begin{tabular}{|c|c|c|c|c|c|c|c|}
\hline $\begin{array}{c}\text { Design } \\
\text { variables }\end{array}$ & $\begin{array}{c}Z_{\max } \cdot 10^{-6} \\
{[\mathrm{~m}]}\end{array}$ & $\begin{array}{c}\theta_{x \max } \cdot 10^{-6} \\
{[\mathrm{rad}]}\end{array}$ & $d_{b}$ & $k_{0}$ & $k_{d}$ & $L_{1}$ & $D_{1}$ \\
\hline \hline 1 & 9.0919 & 22.2955 & 0.301 & 4463.17 & 44.014 & 38.827 & 12.876 \\
\hline 2 & 9.0926 & 22.2847 & 0.296 & 4565.59 & 40.520 & 38.624 & 12.904 \\
\hline 3 & 9.0939 & 22.2627 & 0.318 & 4509.77 & 42.788 & 38.304 & 12.950 \\
\hline 4 & 9.0953 & 22.2549 & 0.317 & 4562.48 & 43.522 & 38.142 & 12.902 \\
\hline 5 & 9.0972 & 22.2264 & 0.315 & 4551.81 & 41.646 & 37.685 & 12.949 \\
\hline 6 & 9.0985 & 22.2117 & 0.322 & 4439.63 & 42.768 & 37.440 & 12.951 \\
\hline 7 & 9.0993 & 22.2085 & 0.311 & 4540.33 & 44.281 & 37.428 & 12.881 \\
\hline 8 & 9.1005 & 22.2061 & 0.313 & 4467.43 & 40.897 & 37.385 & 12.787 \\
\hline
\end{tabular}

To choose the best design from the set of found solutions, it suffices to favor certain criteria compared to others. For example, if the priority is given to minimize the displacement $Z$, the characteristics of the best design are given by $d_{b}=0.301 \mathrm{~mm}, k_{0}=4463.17, k_{d}=44.014$, 
$L_{1}=38.827 \mathrm{~mm}$ and $D_{1}=12.876 \mathrm{~mm}$. However, if the priority is given to minimize the rotational movement $\theta_{x}$, the optimal solution in this case is: $d_{b}=0.313 \mathrm{~mm}, k_{0}=4467.43, k_{d}=40.897$, $L_{1}=37.385 \mathrm{~mm}$ and $D_{1}=12.787 \mathrm{~mm}$.

In order to prove the choice of the optimal values of the design parameters of the electromagnetic spindle for the two selected configuration, we present in Figs. 5 and 6 a comparison of the displacement $Z$ and the rotational movement $\theta_{x}$ obtained with the optimal values and that with initial values indicated in Table 3 . So, according to these two figures, the displacement $Z$ and the rotational movement $\theta_{x}$ are minimum compared to that plotted with the preliminary values of the parameters $d_{b}, k_{0}, k_{d}, L_{1}$ and $D_{1}$.

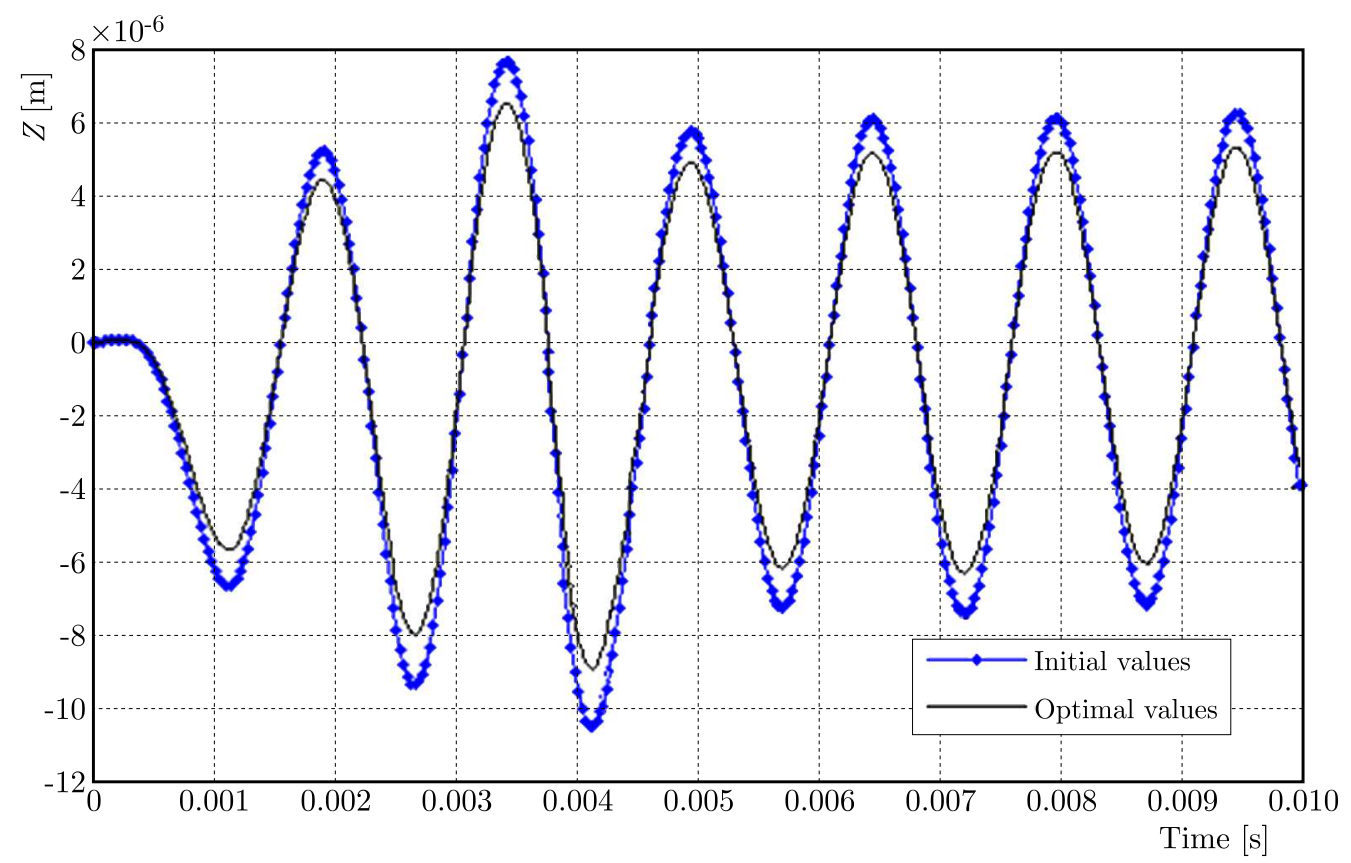

Fig. 6. Comparison of displacement $Z$ obtained with the initial values to that calculated by the optimal values

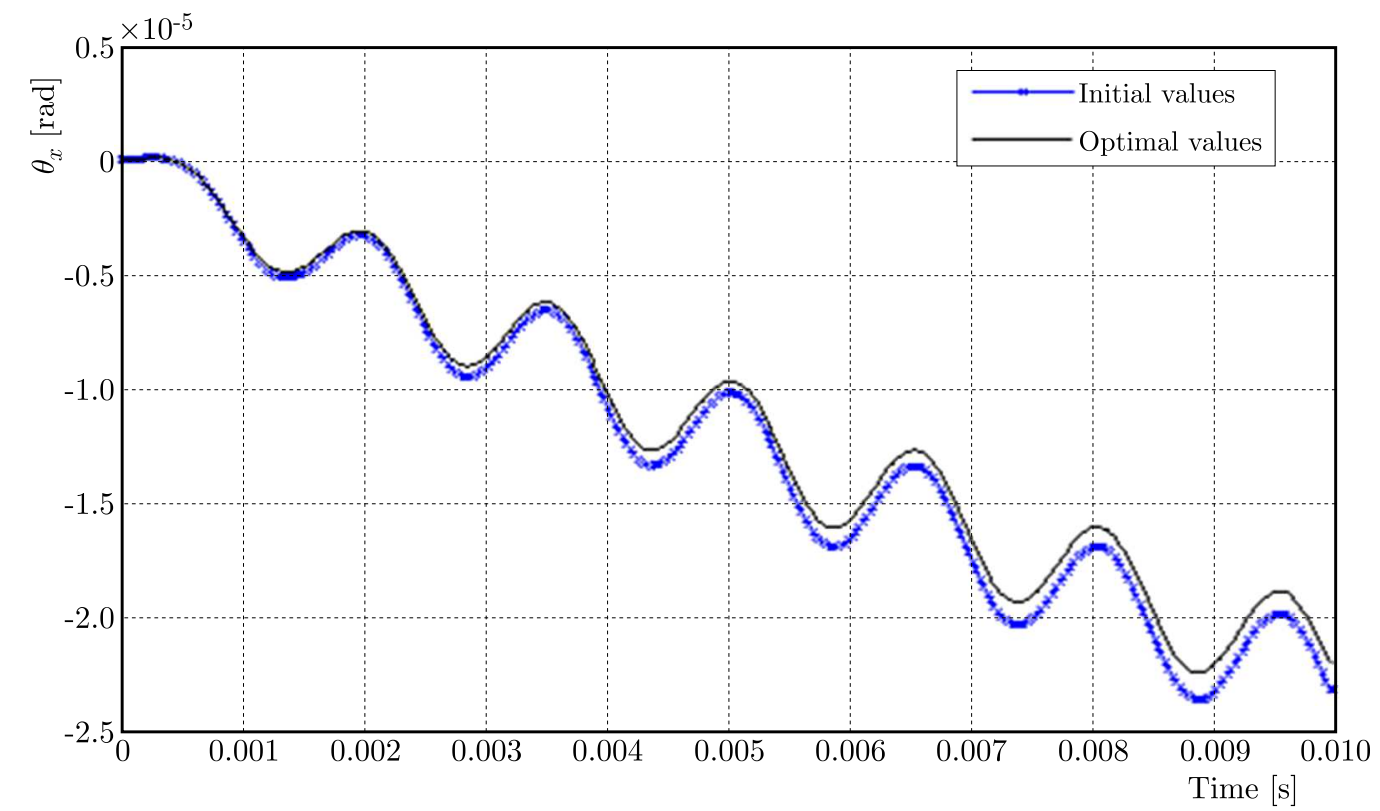

Fig. 7. Comparison of rotational movement $\theta_{x}$ obtained with the initial values to that calculated by the optimal values 


\section{Conclusion}

From the previous study of the electromagnetic spindle system, the validity of interval based simulation to simulate the dynamic behavior with uncertainties has been proved. However, this type of calculation is orientated to solve a linear ordinary system to make dynamic simulations to a time gain which, in particular, influences on the good progress of the preliminary design phase.

The Interval Based Simulation method provides not only a single evaluation of the system behavior but also a set of performance bounds. The simulation results demonstrate that these performance bounds give a better description of the dynamic behavior of electromagnetic spindle system with uncertain parameters.

\section{References}

1. Affi Z., Badreddine E., Romdhane L., 2007, Advanced mechatronic design using a multi-objective genetic algorithm optimization of a motor-driven four-bar system, Mechatronics, 17, $489-500$

2. Alefeld G., Mayer G., 2000, Interval analysis: theory and applications, Journal of Computational and Applied Mathematics, 121, 421-464

3. Amendola G., Dimino I., Concilio A., Amoroso F., Pecora R., 2017, Preliminary design of an adaptive aileron for the next generation regional aircraft, Journal of Theoretical and Applied Mechanics, 55, 307-316

4. Bouaziz A., 2016, Contribution à l'etude du comportement dynamique d'une machine-outil en presence des suspensions magnetiques, ENIS

5. Bouaziz A., Barkallah M., Bouaziz S., Choley J.Y., Haddar M., 2016, Cutting parameters and vibrations analysis of magnetic bearing spindle in milling process, Journal of Theoretical and Applied Mechanics, 54, 691-703

6. Colton J.S., Ouellette M.P., 1994, A form verification system for the conceptual design of complex mechanical systems, Engineering with Computers, 10, 33-44

7. Deb K., Pratap A., Agarwal S., Meyarivan T., 2002, A fast and elitist multiobjective genetic algorithm: NSGA-II, IEEE Transactions on Evolutionary Computation, 6, 182-197

8. Faroughi S., Lee J., 2015, Analysis of tensegrity structures subject to dynamic loading using a Newmark approach, Journal of Building Engineering, 2, 1-8

9. Gościniak I., Gdawiec K., 2019; Control of dynamics of the modified Newton-Raphson algorithm, Communications in Nonlinear Science and Numerical Simulation, 67, 76-99

10. Gourc E., Seguy S., Arnaud L., 2011, Chatter milling modeling of active magnetic bearing spindle in high-speed domain, International Journal of Machine Tools and Manufacture, 51, 928-936

11. Guizani A., Hammadi M., Choley J.Y., Soriano T., Abbes M.S., Haddar M., 2017, Multi-agent approach based on a design process for the optimization of mechatronic systems, Mechanics and Industry, 18, 507

12. Hansen E., Walster G.W., 2003, Global Optimization Using Interval Analysis: Revised and Expanded, CRC Press

13. He Y., McPhee J., 2005, Multidisciplinary design optimization of mechatronic vehicles with active suspensions, Journal of Sound and Vibration, 283, 217-241

14. Hentati T., Bouaziz A., Bouaziz S., Choley J.Y., Haddar M., 2013, Dynamic behaviour of active magnetic bearings spindle in high-speed domain, International Journal of Mechatronics and Manufacturing Systems, 6, 474-492 
15. Kimman M., Langen H., Schmidt R.M., 2010, A miniature milling spindle with active magnetic bearings, Mechatronics, 20, 224-235

16. Knospe C.R., 2007, Active magnetic bearings for machining applications, Control Engineering Practice, 15, 307-313

17. Makowski M., ZaLewski R., 2015, Vibration analysis for vehicle with vacuum packed particles suspension, Journal of Theoretical and Applied Mechanics, 53, 109-117

18. Teorey T. J., YAng D., Fry J.P., 1986, A logical design methodology for relational databases using the extended entity-relationship model, ACM Computing Surveys (CSUR), 18, 197-222

19. Trabelsi H., Yvars P.A., Louati J., Haddar M., 2015, Evaluation of the effectiveness of the interval computation method to simulate the dynamic behavior of subdefinite system: application on an active suspension system, International Journal on Interactive Design and Manufacturing (IJIDeM), 9, 83-96

20. Vu N.A., Lee J.W., Le T.P.N., Nguyen S.T.T., 2016, A fully automated framework for helicopter rotor blades design and analysis including aerodynamics, structure, and manufacturing, Chinese Journal of Aeronautics, 29, 1602-1617

Manuscript received January 29, 2019; accepted for print April 2, 2019 